\title{
NONDEGENERATE SURFACES OF FINITE TOPOLOGICAL TYPE $\left({ }^{1}\right)$
}

\author{
BY \\ W. H. FLEMING
}

1. Introduction. In the theory of surface area and its applications to the calculus of variations Morrey's theorem plays an important role. The purpose of this paper is to generalize this result to surfaces of the topological type of any compact 2-manifold, orientable or not, with or without boundary:

THEOREM. Every nondegenerate Fréchet surface of any finite topological type $\tau$ with finite Lebesgue area has a quasi-conformal representation $T(w)$. We may take as the domain of $T(w)$ a certain normalized parallel slit domain.

For 2-cells and 2-spheres this is Morrey's theorem [7], also [1, p. 484]. We shall suppose here that $\tau$ is neither of these types. The proof strongly uses the result that a polyhedron of higher topological type has a quasi-conformal representation on a normalized parallel slit domain [3, Chapter II] $\left({ }^{2}\right)$. This replaces a classical result for the 2-cell case due to Schwarz. Slit domains were used by Courant [3] and Shiffman [11] in their work on the Plateau problem. In fact, several lemmas in the present paper have counterparts in [11].

For surfaces defined as mappings from any admissible plane set $[1$, p. 27] Morrey's theorem was used by Cesari in the proof that any such surface with finite Lebesgue area has a generalized conformal representation $[1, \mathrm{p} .487]$ with absolutely continuous plane projections $[1$, p. 544 , the third theorem ]. For surfaces of the type of the 2-cell or 2-sphere Morrey's theorem has been used by Radó [9, especially p. 501] together with cyclic decompositions to prove that the Lebesgue area $L(S)$ equals the lower area defined in [9] provided $L(S)$ is finite. Cesari [1] has since shown by other methods that this equality holds even if $L(S)=\infty$, for surfaces defined as mappings from any admissible plane set. Neugebauer and the author plan to give a new proof of Cesari's result (under the assumption $L(S)<\infty$ ) based on the above extension of Morrey's theorem and Cesari's fine cyclic decompositions of surfaces [2]. The fine cyclic elements may be slightly degenerate and need not have any quasi-conformal representation (see §8). However, any fine cyclic element becomes nondegenerate upon cutting it at a finite number of points at most.

Presented to the Society November 23, 1956; received by the editors March 26, 1957.

(1) This research was supported by ARDC under contract AF 18(600)-1484 at Purdue University.

(2) In place of slit domains, various other kinds of domains could be used. See $\S 8$ and references cited there. 
Methods similar to the ones in this paper are used by the author elsewhere (to appear) to obtain representations of generalized surfaces of finite topological types (see Young [13]), and thereby in connection with parametric problems of the calculus of variations.

\section{PURELY TOPOLOGICAL PART}

2. Definitions and preliminaries. The letter $A$ will stand for a 2-manifold $[14$, p. 25], $w$ a point of $A$, and $T(w)$ a continuous mapping from $A$ to euclidean $N$-space $E_{N}(N \geqq 2)$. $A$ may be closed, or may have disjoint boundary curves $C_{1}, \cdots, C_{p}$. Let $q$ denote the characteristic of $A$. If $A$ is orientable, then $A$ is a topological sphere with $\nu$ holes and $h$ handles, where

$$
h=(q-\nu+2) / 2 .
$$

In the nonorientable case, $A$ is a sphere with $\nu$ holes and $k=q-\nu+2$ cross- $^{-}$ caps. The quantity $\tau=(\nu, q, \epsilon)$, where $\epsilon$ denotes orientability or not, will be called topological type of $A$. The boundary $C_{1} \cup \cdots \cup C_{\nu}$ of $A$ is denoted by $A^{*}$. If $B$ is a subset of $A, B^{0}$ and $B^{-}$denote, respectively, its interior and closure relative to $A$. We use the term relative boundary of $B$ for $B^{-} \cap(A-B)^{-}$.

By cross-cut we mean an arc in $A$ with endpoints on $A^{*}$ not meeting $A^{*}$ between endpoints. Let $\Omega(A)$ denote the set of all closed curves in $A$ not null homotopic, together with all cross-cuts $\gamma$ such that there is no $\operatorname{arc} \lambda$ of $A^{*}$ with the same endpoints as $\gamma$ such that $\gamma \cup \lambda$ is null homotopic. Clearly every cross-cut with endpoints on different boundary curves $C_{i}, C_{j}$ belongs to $\Omega(A)$. The following known result will often be used:

Lemma 1. A simple closed curve $\gamma$ in $A$ is null homotopic if and only if $\gamma$ bounds a 2-cell $Z \subset A$.

In particular, every simple closed curve or cross-cut $\gamma$ which does not separate $A$ belongs to $\Omega(A)$, unless $A$ is a 2-cell and $\gamma=A^{*}$.

Let $S$ denote a Fréchet surface in $E_{N}$, of type $\tau$. We consider only nonoriented surfaces. However, if $\tau$ is an orientable type one of the two possible orientations may be assigned and the discussion still applies with only minor modifications. $\left\|S, S^{\prime}\right\|$ denotes Fréchet distance, and is of course only defined if $S$ and $S^{\prime}$ are of the same topological type.

For brevity we say $(T, A)$ represents $S$, or simply $T$ represents $S$, to mean $T$ is a representation of $S$ with domain $A$. Let $\Gamma(T, A)$ denote the set of all maximal continua of constancy of $T$.

(2.1) Definition. A surface $S$ is nondegenerate if, for every representation $(T, A)$ of $S$ :

(a) Given $g \in \Gamma(T, A)$ there exists an open set $G$ containing $g$ such that no $\gamma \in \Omega(A)$ is entirely contained in $G$;

(b) No $g \in \Gamma(T, A)$ separates $A$ (i.e., $A-g$ is connected for every $g$ ); and

(c) $T$ is not constant on $A^{*}$; or, if $A$ is closed, $T$ is not constant on $A$. 
For 2-cells and 2-spheres this definition agrees with that of open and closed nondegenerate surface, respectively, given by Cesari [1, p. 472]. If $S$ is neither of these types (a) implies (c). For each $\epsilon>0$ let $\Phi(\epsilon)$ denote the set of all continua $K$ separating $A$ such that diam $T(G)>\epsilon$ for at least two components $G$ of $A-K$. Let

$$
\begin{aligned}
\phi(S) & =\inf _{\gamma \in \Omega(A)} \operatorname{diam} T(\gamma), \\
\psi(S, \epsilon) & =\inf _{K \in \Phi(\epsilon)} \operatorname{diam} T(K),
\end{aligned}
$$

where $(T, A)$ is any representation of $S$.

If $\Omega(A)$ is empty we set $\phi(S)=+\infty$, and similarly if $\Phi(\epsilon)$ is empty then $\psi(S, \epsilon)=+\infty$. The indicated Fréchet invariance is easily verified. $\phi(S)$ is continuous. This will be needed later, and also the useful inequality:

$$
\psi(S, \epsilon-\sigma) \leqq \psi\left(S^{\prime}, \epsilon\right)+\sigma, \text { if }\left\|S, S^{\prime}\right\|<\sigma / 2 .
$$

Lemma 2. $S$ is nondegenerate if and only if $\phi(S)>0, \psi(S, \epsilon)>0$ for every $\epsilon>0$, and in case $S$ is of the type of the 2-cell or 2-sphere (2.1c) holds.

Proof. Necessity. The proof that $\psi(S, \epsilon)>0$ is essentially the same as for the special case $[1,33.4(\mathrm{i})]$. If we assume $\phi(S)=0$, then by Zoretti's theorem $[1,(10.2)]$ there exist $\gamma_{n} \in \Omega(A)$ and a continuum $K$ on which $T$ is constant such that infinitely many $\gamma_{n}$ belong to any open set containing $K$. This is contrary to (2.1 a). Sufficiency. Given $g$ take in $(2.1 \mathrm{a}) \mathrm{G}$ as the set of $w$ for which $|T(w)-T(g)|<\phi(S)$. Obviously $(2.1 \mathrm{~b})$ holds, and except when $A$ is a 2-cell or 2-sphere (a) implies (c).

3. Continuation. The following theorem, taken together with an important approximation theorem of J. W. T. Youngs [15] proves that $S$ is nondegenerate if and only if $S$ has a light representation. Therefore, our definition is equivalent to that used by Morrey [7] and Radó [9]. This theorem will not be used in proving the main result concerning quasi-conformal representations.

TheOREM. A surface $S$ is nondegenerate of type $\tau$ if and only if its middle space is a 2-manifold of type $\tau$.

Proof. Sufficiency. This is immediate from the fact that $S$ has a light representation if $A$ and $M$ are homeomorphic, which follows from Youngs' approximation theorem just cited.

Necessity. Suppose first that $A$ is closed. By results of Roberts and Steen$\operatorname{rod}[10]$, see also Youngs $[14,(6.4)$ and (6.5)], $M$ is homeomorphic with $A$ if (and only if) for every $g \in \Gamma$ and open set $G$ containing $g$ there exists a 2-cell $Z$ such that $g \subset Z^{0}$ and $Z \subset G$. Consider $g \in \Gamma$ and $G$ an open set containing $g$. Using (a) let $G^{\prime}$ be an open subset of $G$ such that $g \subset G^{\prime}$ and every closed curve in $G^{\prime}$ is null homotopic in $A$. By (b), $U=A-g$ is connected. By a lemma of Roberts and Steenrod $[10 ; 14]$ there exist disjoint simple 
closed curves $\lambda_{1}, \cdots, \lambda_{m}$ contained in $G^{\prime}-g$ such that $\lambda_{1} \cup \cdots \cup \lambda_{m}$ bounds a 2-manifold $B$ contained in $U$ and for each $i=1, \cdots, m$ there is an open cylinder $B_{i}$, bounded by $\lambda_{i}$ and a portion of $g$, such that $B_{i}^{-} \subset G^{\prime}$. The set $Z=A-B=B_{1} \cup \ldots \cup B_{m} \cup g$ is connected, since each point of $Z$ can be joined in $Z$ to the continuum $g$. Then $m=1$; otherwise, since both $Z$ and $B$ are connected, no single $\lambda_{i}$ separates $A$. Then $\lambda_{i} \subset G^{\prime}$ is not null homotopic in $A$, contrary to choice of $G^{\prime}$. Similarly, since $Z \subset G^{\prime}$ every simple closed curve in $Z$ separates $A$, therefore also separates the open set $Z$. Thus $Z$ is an open 2-cell bounded by $\lambda_{1}$. Clearly, $g \subset Z$ and $Z^{-} \subset G$. Since $g$ and $G$ were chosen arbitrarily, $M$ is homeomorphic with $A\left(^{3}\right)$.

Before considering surfaces with boundary we prove the following:

Lemma 3. Let $(T, A)$ represent a nondegenerate surface $S$. Then for any $g \in \Gamma, g \cap A^{*}$ is either void, a point, or an arc.

Proof. Since no $g$ contains any component of $A^{*}$, it suffices to show that $g \cap A^{*}$ is connected or void for any $g$. Suppose there were two points $w_{1}$ and $w_{2}$ belonging to different components of $g \cap A^{*}$. By known theorems: given $\delta>0$, $w_{1}$ and $w_{2}$ can be joined by a cross-cut $\gamma$ such that diam $T(\gamma)<\delta$. If $\delta<\phi(S)$, then $\gamma$ together with an arc $\lambda$ of a single component $C_{i}$ of $A^{*}$ bound a 2-cell by Lemmas 1 and 2. Choose $\epsilon=\min \left[\operatorname{diam} T(\lambda), \operatorname{diam} T\left(C_{i}-\lambda\right)\right] / 2$, and $\delta<\psi(S, \boldsymbol{\epsilon})$. By Lemma 2 this is a contradiction.

Now let $A$ have boundaries $C_{1}, \cdots, C_{\nu}$; let $A^{\prime}$ be a closed 2-manifold obtained by capping each boundary $C_{i}$ with an open 2-cell $Z_{i}$ [14]. Let $\Gamma^{\prime}$ denote the upper semi-continuous collection of continua consisting of the elements of $\Gamma$ and points of $Z_{i}$ for $i=1, \cdots, \nu$. Let $T_{1}$ be the monotone mapping carrying each $w \in A^{\prime}$ onto that $g \in \Gamma^{\prime}$ containing $w$, and $M^{\prime}$ the set $\Gamma^{\prime}$ topologized so that $T_{1}$ is continuous. We may regard $M$ as $T_{1}(A) . M^{\prime}$ does not reduce to a point. Let us verify (2.1) (a) and (b) for every $g \in \Gamma^{\prime}$. They obviously hold if $g \in \Gamma^{\prime}-\Gamma$. Consider $g \in \Gamma ; g$ does not separate $A^{\prime}$, since $g$ neither separates $A$ nor contains any boundary $C_{j}$. Let $G$ open in $A$ be as in (2.1 a). If $G \cap A^{*}$ is void, set $G^{\prime}=G$. Otherwise, using Lemma 3 there is a set $G_{1}$ open in $A$ such that $g \subset G_{1} \subset G$ and $G_{1} \cap A^{*}$ is an arc of a single $C_{i}$. Let $G^{\prime}$ be an open subset of $A^{\prime}$ obtained by adjoining to $G_{1}$ a simply connected part of $Z_{i}$ bounded by $G_{1} \cap C_{i}$ and an arc in $Z_{\bar{i}}$. Every closed curve in $G^{\prime}$ is homotopic to a closed curve in $G_{1}$, and so is null homotopic in $A^{\prime}$. This shows that $\Gamma^{\prime}$ satisfies (a) and (b). Therefore, $A^{\prime}$ and $M^{\prime}$ are homeomorphic.

The restriction of $T_{1}$ to $Z_{\bar{j}}$ is one-one on $Z_{j}$ for each $j$; and by Lemma 3, monotone on $Z_{j}^{-}$, not constant on $Z_{j}^{*}$. By $\left[9\right.$, p. 65] $T_{1}\left(Z_{j}^{-}\right)$is a 2 -cell for each $j$, and by Lemma 3 these 2 -cells are disjoint. This implies that $M$ is a 2-manifold of the same topological type as $A$.

(3) What has actually been shown is that if $M$ is the image of a closed 2-manifold $A$ under a monotone mapping $T^{\prime}, \Gamma\left(T^{\prime}, A\right)$ satisfies (2.1) (a) and (b), and $M$ is not a point, then $M$ and $A$ are homeomorphic. This remark is used below 


\section{ANALYTICAL PART}

4. Preliminaries. I write $a(P)$ for the elementary area of a polyhedron $P$ and $L(S)$ for the Lebesgue area of a surface $S$, defined as the lower limit of elementary areas of nondegenerate polyhedra tending to $S$.

For the remainder of the paper we shall suppose $A$ is a parallel slit domain (see §5), since this class will suffice for the problem of quasi-conformal representation. For the closed case $(\nu=0), A$ will consist of the full plane including a point at $\infty$ with a finite number of semi-infinite identified slits parallel to the real axis; whereas for $\nu \geqq 1 A$ is an upper half plane with a finite number of slits of a similar sort.

It is convenient to represent any finite point of $A$ by its $(u, v)$ coordinates. On the slits the correspondence between points of $A$ and points $(u, v)$ is not biunique, but is determined by the identifications. A continuous mapping from $A$ to $E_{N}$ is simply any vector function $T(u, v)=\left[T^{1}(u, v), \cdots, T^{N}(u, v)\right]$ which is continuous in every closed region whose interior intersects no slit, takes the same value at identified points on the slits, and is continuous at $\infty$. We write

$$
D(T, A)=\frac{1}{2} \iint(E+G) d u d v, \quad I(T, A)=\iint\left(E G-F^{2}\right)^{1 / 2} d u d v
$$

for the Dirichlet integral and classical area integral, respectively, whenever they exist. The integrals are taken over the full plane or upper half plane according as $A$ is closed or not.

$T(u, v)$ is called a Dirichlet mapping if: (1) $T(u, v)$ defines a continuous mapping from $A$ into $E_{N}$; (2) each component $T^{i}(u, v)$ is absolutely continuous in Tonelli's sense in every closed $(u, v)$ interval disjoint from the slits of $A, i=1, \cdots, N$; and $(3) D(T, A)$ is finite. $T(u, v)$ is quasi-conformal $\left({ }^{4}\right)$ if $T(u, v)$ is Dirichlet and $D(T, A)=I(T, A)$. The latter is equivalent to $E=G$, $F=0$ almost everywhere.

5. Parallel slit domains. This section follows, in part, Shiffman [11].

(a) Closed slit domains. In a $(u, v)$ plane let there be made $\beta(\geqq 2)$ slits parallel to the $u$-axis, semi-infinite to the right. The ordinates $V_{j}$ of the slits are ordered so that $V_{1}<\cdots<V_{\beta}$. The two sides of each slit are regarded as distinct. There are also given critical abscissae $U_{1}<\cdots<U_{\alpha}(\alpha \geqq 1)$. Let $F$ denote the set of points $\left(U_{i}, V_{j}\right), i=1, \cdots, \alpha, j=1, \cdots, \beta$. The left endpoint of every slit shall belong to $F$, and $F$ generally contains other points as well. Identifications are made as follows. Let $s$ be any segment on a slit $L$, with endpoints in $F$ or one endpoint in $F$ and the other $\infty$. Then $s$ is identified with exactly one other segment $s^{\prime}$, on another slit or on the opposite side of $L$, whose endpoints have the same abscissae as $s$. In fact, the identifi-

(4) This term has been used by L. C. Young [12]. It is equivalent to the term almost conformal used by Cesari $[1, \mathrm{p} .477]$, and to the term generalized conformal used by Rad6 $[9, \mathrm{p}$. 480] and Morrey [7]. 
cation of $s$ and $s^{\prime}$ is made pointwise, each pair of identified points having the same abscissa $\left({ }^{5}\right)$. To insure that $\infty$ belongs to an open 2 -cell, we must require that the identifications for abscissa $u$ in the interval $\left(U_{\alpha}, \infty\right)$ suitably "interlock" [11].

This defines a closed slit domain $A$. The order of a finite point $w$ on the slits is $a+b / 2-1$, where $a$ is the number of left endpoints of slits and $b$ the number of other points corresponding to $w$. Those points with positive order are called vertices of $A$. A pair of identified segments joining vertices, or a vertex with $\infty$, define an edge. Clearly every vertex has critical abscissa.

(b) Slit domains with $\nu(\geqq 1)$ boundaries. The following modifications in (a) are needed. We consider the upper half plane with $\beta(\geqq 1)$ semi-infinite slits with positive ordinates $V_{j}$ and critical abscissae $U_{i}$, as before. $F$ consists of the points $\left(U_{i}, V_{j}\right), i=1, \cdots, \alpha, j=0,1, \cdots, \beta$, where we set $V_{0}=0$. A segment $s$ may be identified with another segment on a slit as before, or with a segment on the real axis lying directly below $s$; or $s$ may be unidentified. The unidentified segments of slits, together with the unidentified part of the real axis form the boundary $A^{*}$. Vertices not on $A^{*}$ are defined as before, and are called inner. The order of a finite point $w \in A^{*}$ is $a+b / 2-1 / 2$, and a point of $A^{*}$ with positive order is a boundary vertex. Since at least part of the negative real axis is unidentified, $\infty$ is on $A^{*}$. The identifications for $u$ in the interval $\left(U_{\alpha}, \infty\right)$ must be such that $\infty$ belongs to a half-open 2-cell. A free edge is a segment of a slit, or of the real axis, joining boundary vertices or a boundary vertex with $\infty$. If no part of the real axis is identified, it counts as a free edge. Every boundary vertex adjoins exactly two free edges.

A slit domain $A$ is called normalized if: (1) The left endpoint of every slit corresponds to a vertex and every critical abscissa $U_{i}$ is the abscissa of at least one vertex; (2) $U_{1}=0, V_{\beta}=1$, and if $A$ is closed $V_{1}=0\left(^{6}\right)$. Condition (1) excludes superfluous abscissae $U_{i}$ as well as the possibility that every point of a slit $L$, or of a segment at the left end of $L$, is merely identified with the point on the opposite side of $L$. Any slit domain, not topologically a 2 -sphere or 2-cell, can be normalized by deleting such superfluous abscissae and slits (or pieces of slits) followed by a linear transformation of the complex plane (or upper half plane) onto itself.

It is not excluded that some point $p$ on $L$ to the right of a vertex is identified with that point of $L$ opposite $p$. For example, $L$ may carry two free edges situated on opposite sides of a same finite segment $t$ of $L$, every point of $L$ to the right of $t$ being identified with the point opposite.

(c) Convergence of normalized slit domains; (cf. [11, p. 861]). Let us say that two normalized slit domains are similarly arranged if the geometric configuration of the slits, vertices, and edges (as well as the manner of identifying

(5) This condition arises naturally in mapping a closed Riemann domain conformally onto a slit domain [3, Chapter 2].

${ }^{(6)}$ This normalization replaces the 3-point condition used in proving Morrey's Theorem. 
edges) is the same for both. Similarly arranged slit domains obviously are of the same topological type $\tau$, but not conversely. If $q$ denotes the characteristic, the sum of the orders of the vertices is $q+2$ or $q+1$ according as $A$ is closed or not $[11$, pp. $858-859]$. It follows that there are only finitely many different arrangements for domains of any given type $\tau$.

By distance between two finite points $w, w^{\prime}$ of a slit domain $A$ we mean the euclidean length of the shortest curve in $A$ joining $w$ and $w^{\prime}$.

Let $A_{1}, A_{2}, \cdots$ be normalized slit domains, similarly arranged, such that corresponding points of $F_{n}$ tend to finite limits as $n \rightarrow \infty$. The limit domain $A$ is defined as follows. $F$ is the set of limit points of $F_{n}$; i.e., the slits and critical abscissae of $A$ are the limits of the slits and critical abscissae, respectively, of $A_{n}$. Several points of $F_{n}$ may tend to the same point of $F$. The identifications in $A$ are determined if we specify them at any point $p$ on a slit whose abscissa $u$ is not critical. If $p$ is on the upper (lower) side of a slit $L$, for large $n$ let $p_{n}$ be that point with abscissa $u$ on the upper side of the highest slit (lower side of the lowest slit) of $A_{n}$ which tends to $L . p \in A^{*}$ if the distance in $A_{n}$ between $p_{n}$ and $A_{n}^{*}$ tends to 0 as $n \rightarrow \infty$. $p$ is identified with another point $p^{\prime}$ with abscissa $u$ if the distance in $A_{n}$ between $p_{n}$ and $p_{n}^{\prime}$ tends to 0 .

With these definitions $A$ is a slit domain, not necessarily normalized, possibly with fewer boundaries or lower characteristic than $A_{n}$. We shall next determine conditions under which $A$ and $A_{n}$ are homeomorphic.

(d) Some curves in $\Omega(A)$. If $A$ is a normalized slit domain the following types of curves belong to $\Omega(A)$ :

(5.1) A closed curve or cross-cut lying entirely on a finite portion of a single vertical line. No such curve separates $A$.

(5.2) A component $C_{i}$ of $A^{*}$ (we assume $A$ is not a 2-cell).

(5.3) A simple closed curve $\gamma$ formed by joining identified points $p$ and $p^{\prime}$ by an arc not meeting any slit between endpoints nor passing through $\infty$. If $p$ and $p^{\prime}$ are on different slits, $\gamma$ does not separate $A$. If $\gamma$ separates, then each component of $A-\gamma$ must contain a component of $A^{*}$ and we apply Lemma 1.

(5.4) A simple closed curve formed by joining identified points $p$ and $p^{\prime}$ on an identified edge whose right endpoint is a vertex (i.e., not $\infty$ ) with $\infty$ by a pair of arcs meeting no slit between endpoints. See [11, p. 859].

(5.5) A simple closed curve $\gamma$ passing through $\infty$ encircling at least one slit (but not all slits if $A$ is closed) and meeting no slit at any finite point. Either $\gamma$ does not separate; or if it does, each component of $A-\gamma$ contains in its closure a closed curve of one of the previous types.

(e) Dissection of slit domains by gratings. Let $\pi$ be a grating composed of the perimeter $R^{*}$ of an interval $R$ in the $u, v$ plane together with a finite number of segments parallel to the coordinate axes with endpoints on $R^{*}$. We say that $\pi$ is based on $R .[\pi]$ is the set in the plane occupied by $\pi . \Delta(\pi)$, $\bar{\Delta}(\pi)$ denote, respectively, the minimum and maximum distance between 
adjacent parallel lines of $\pi$. Let $A$ be a slit domain whose slits are disjoint from the horizontal lines of $\pi$ and whose critical abscissae are distinct from the abscissae of vertical lines of $\pi$. $K$ denotes the set of points in $A$ with $(u, v)$ coordinates in $[\pi]$. We say that $\pi$ is fine relative to $A$ if $F \subset R$ and $2 \bar{\Delta}(\pi)$ is less than $\min \left(U_{i+1}-U_{i}\right)$ and $\min \left(V_{j+1}-V_{j}\right)$. If $\pi$ is fine every component of $A-K$ is an open or half open 2-cell. If $A$ carries a subscript $n$ the sets $F$ and $K$ will carry that same subscript.

In Lemma 4 (as well as certain later sections) the following notation applies. Let $A_{n}$ tend to $A$, and $\pi$ a grating fine relative to $A$. We dissect both $A$ and $A_{n}$ with $\pi$, for large $n$. The grating $\pi$ need not be fine relative to $A_{n}$; see examples in [3] and [11]. $d>0$ is a number not exceeding the distance between $[\pi]$ and $F$. Let $n(d)$ be such that the set $F_{n}$ is in the $d / 2$ neighborhood of $F$ for every $n \geqq n(d)$. In particular, for $n \geqq n(d)$ every slit of $A_{n}$ is distant at most $d / 2$ from some slit of $A . Z_{1}, \cdots, Z_{k}$ are the components of $A-K$; $\gamma_{j}=Z_{j}^{*} \cap K$ the relative boundary of $Z_{j} ; w_{j}=\left(u_{j}, v_{j}\right)$ a point of $\gamma_{j}$ distant at least $d / 2$ from any slit of $A$; and $w_{j n}$ the point of $A_{n}$ with coordinates $\left(u_{j}, v_{j}\right)$, $j=1, \cdots, k$.

Lemma 4. Choose $n \geqq n(d)$. Suppose that, if $Z$ is any component of $A_{n}-K_{n}$, then its relative boundary $Z^{*} \cap K_{n}$ contains no simple closed curve or cross-cut $\gamma \in \Omega\left(A_{n}\right)$. Then there is a homeomorphism $H_{n}$ of $A$ onto $A_{n}$ such that $H_{n}\left(w_{j}\right)$ $=w_{j n}$ and $H_{n}\left(Z_{j}\right)$ is a component of $A_{n}-K_{n}$ for $j=1, \cdots, k$. In particular, $A$ and $A_{n}$ are of the same topological type.

Proof. Let $\gamma_{j n}$ denote the following simple closed curve or cross-cut lying in $K_{n}$, homeomorphic with $\gamma_{j}$. Outside the $d / 2$ neighborhood of the slits of $A, \gamma_{j n}$ and $\gamma_{j}$ are superimposed. Each piece $\lambda$ of the intersection of $\gamma_{j}$ with the $d / 2$ neighborhood is either a vertical line ending at a point $p$ on the boundary $A^{*}$ or a pair of vertical lines, one ending at a point $p$ on an identified edge, the other at the point $p^{\prime}$ identified with $p$. In the first case the corresponding $\lambda_{n}$ is a vertical curve with the same starting point as $\lambda$ which proceeds to $p_{n}$ (notation of (c) above) and then joins $p_{n}$ following the identifications in $A_{n}$ to $A_{n}^{*}$. The second case is similar.

Clearly $\gamma_{j n}$ is all or part of the relative boundary of some component of $A_{n}-K_{n}$. Then by hypothesis and Lemma $1, \gamma_{j n}$ separates $A_{n}$ and moreover one of the two components of $A_{n}-\gamma_{j n}$ is an open or half-open 2-cell. Let $Z_{j n}$ be that component corresponding naturally to $Z_{j}$, i.e., superimposed on $Z_{j}$ except at most in the $d / 2$ neighborhood of the slits of $A . Z_{1 n}, \cdots, Z_{k n}$ are disjoint since $\gamma_{i n} \neq \gamma_{j n}$ for $i \neq j$. The complement of $Z_{j n}$ contains a closed curve of type (5.3) or (5.5) according as $Z_{j n}$ contains $\infty$ or not, since $\pi$ is fine relative to $A$. Hence, $Z_{j n}^{-}$is a 2 -cell for each $j$. Moreover, there are no other components of $A_{n}-K_{n}$ since any such component must contain in its relative boundary a curve of type $(5.1)$; cf. [11, p. 862].

We define $H_{n}$ first as a homeomorphism from $K$ onto $K_{n}$, such that 
$H_{n}\left(\gamma_{j}\right)=\gamma_{j n}$ and $H_{n}\left(w_{j}\right)=w_{j n}, j=1, \cdots, k$. By a known theorem $[1$, p. 55] $H_{n}$ has an extension as a homeomorphism from $Z_{j}^{-}$onto $Z_{j n}^{-}$for $j=1, \cdots, k$.

6. Continuation. Let $Q_{a}$ denote the square with center the origin, side length $a$, and sides parallel to the $u, v$ axes. The following is a variant of an important lemma due to L. C. Young [12, p. 318]; the proof is almost the same as Young's.

Lemma 5 ( $\epsilon \delta$ grating). Given $M>0$ and $\epsilon>0$ there exists $\eta=\eta(M, \epsilon)>0$ with the following property. If $T(u, v)$ is any Dirichlet mapping from a slit domain $A$ into $E_{N}$ with $D(T, A) \leqq M$ and $a>0$ is arbitrary, there exists $\delta$ with $\eta<\delta<\epsilon$ and a grating $\pi$ based on $Q_{a}$ such that $a \delta<\Delta(\pi) \leqq \bar{\Delta}(\pi)<2 a \delta$ and, if $\gamma$ $i$ any vertical or horizontal side not on $Q_{a}^{*}$ of a rectangle formed by the grating $\pi$, then $T$ represents on $\gamma$ a finite sum of curves of total length $<\epsilon$.

Let $T_{n}$ be defined in the slit domain $A_{n}$ for $n=1,2, \cdots$. We call the sequence $T_{n}$ equicontinuous if: (1) for every $a>0$ and $\epsilon>0$ there exists $\delta>0$ such that $\left|T_{n}(w)-T_{n}\left(w^{\prime}\right)\right|<\epsilon$ if $w$ and $w^{\prime}$ are distant in $A_{n}$ (cf. $\left.\S 5(\mathrm{c})\right)$ less than $\delta$ and both $w$ and $w^{\prime}$ lie inside the square $Q_{a}, n=1,2, \ldots$; and (2) given $\epsilon>0$ there exists $a>0$ such that $\left|T_{n}(w)-T_{n}(\infty)\right|<\epsilon$ for all $w$ outside $Q_{a}$ and $n=1,2, \cdots$ (equicontinuity at $\infty$ ).

Lemma 6. Let $S_{n}$ tend to a nondegenerate surface $S$, and let $T_{n}(u, v)$ be a Dirichlet representation of $S_{n}$ on a normalized slit domain $A_{n}$ with $D\left(T_{n}, A_{n}\right)$ bounded by a constant $M$ independent of $n$. Then there is a subsequence of $n$ for which $A_{n}$ tends to a limit $A$ homeomorphic with $A_{n}$ and $T_{n}$ is equicontinuous.

Proof. For definiteness suppose $A_{n}$ has boundary. If $A_{n}$ is closed the proof is similar and slightly simpler. There are only finitely many possible arrangements of $A_{n}$, all $A_{n}$ being of the same topological type. We may select a subsequence (denoted again by $T_{n}, A_{n}$ ) for which all $A_{n}$ are similarly arranged and corresponding points of $F_{n}$ tend to finite limits or to $\infty$.

Let us first show that no points of $F_{n}$ tend to $\infty$. Choose positive

$$
\epsilon<\phi(S) / 24
$$

(see Lemma 2) and $\eta=\eta(M, \epsilon)$. Let $a$ be chosen so that all finite limit points of $F_{n}$ are interior to the square $Q_{\eta a}$. Choose $n_{0}$ so that for $n \geqq n_{0}, \phi\left(S_{n}\right)$ $\geqq \phi(S) / 2$ and each point of $F_{n}$ either lies inside $Q_{\eta a}$ or outside $Q_{a}$. Consider fixed $n \geqq n_{0}$ and $\epsilon \delta$ grating $\pi$ based on $Q_{a}$ with $T(u, v)=T_{n}(u, v)$ and $A=A_{n}$ in Lemma 5. Let $R$ be a rectangle composed of a rectangle $R^{\prime}$ formed by $\pi$ whose closure contains the origin, together with the 8 rectangles contiguous to $R^{\prime} . Q_{\eta_{a}} \subset R$ and $R \subset Q_{a}$ if $\epsilon<1 / 8$. Let $B_{n}$ be the part of $A_{n}$ inside $R$ and $\gamma_{n}$ the relative boundary of $B_{n}$. The $T_{n}$ image of each component of $\gamma_{n}$ has diameter $<12 \epsilon<\phi\left(S_{n}\right)$; therefore no such component belongs to $\Omega\left(A_{n}\right)$. It follows that $\gamma_{n}$ is a cross-cut with both endpoints on that component of $A_{n}^{*}$ containing $\infty\left(\gamma_{n}\right.$ certainly meets this component on the negative real 
axis since all slits are in the right half plane). By Lemma 1 either $B_{n}^{-}$or $A_{n}-B_{n}$ is a 2 -cell.

Since $A_{n}$ is normalized, $B_{n}$ contains at least one vertex $w . B_{n}$ meets some identified edge; otherwise $w$ is the left endpoint of two free edges lying on opposite sides of the same slit and both meeting $\gamma_{n}$. Since $\gamma_{n}$ is a cross-cut also meeting $A_{n}^{*}$ on the negative real axis this is impossible. Then $B_{n}$ contains a closed curve of type (5.3), which proves that $A_{n}-B_{n}$ must be a 2-cell. Therefore, $A_{n}-B_{n}$ contains no curve of type (5.4), which implies that no point of $F_{n}$ is in $A_{n}-B_{n}$. Thus, all points of $F_{n}$ tend to finite limits and the limit domain $A$ is defined. .

Let us next show that $A$ and $A_{n}$ are homeomorphic. Let $\beta$ denote the number of slits of $A$. Choose $a$ so that $F \subset Q_{a / 2}$, and $\epsilon<\phi(S) / 24(\beta+1)$ small enough that any grating $\pi$ based on $Q_{a}$ with $\bar{\Delta}(\pi)<6 \epsilon a$ is fine relative to $A$. Let $\eta_{1}=\eta(M, \epsilon)$ and $d=\eta_{1} a$. Fix $n \geqq n(d)$ large enough that $\phi\left(S_{n}\right) \geqq \phi(S) / 2$ and let $\pi_{0}$ be an $\epsilon \delta$ grating based on $Q_{a}$ with $T=T_{n}$ and $A=A_{n}$ in Lemma 5 . Let $\pi$ be a subgrating obtained by deleting all lines in $\pi_{0}$ distant at most $d$ from $F$ together with part of $\pi_{0}$ in a certain neighborhood of $Q_{a}^{*}$. Then $\bar{\Delta}(\pi)$ $<6 \delta a<6 \epsilon a$.

The unbounded component of $A_{n}-K_{n}$ is a 2 -cell with a cross-cut as relative boundary. Every bounded component not meeting any slit is a rectangle. If $Z$ is any bounded component meeting some slit, and $\gamma$ any component of the relative boundary of $Z$, then

$$
\operatorname{diam} T_{n}(\gamma)<12(\beta+1) \epsilon<\phi\left(S_{n}\right) .
$$

Hence, $\gamma \notin \Omega\left(A_{n}\right)$. By Lemma $4, A$ and $A_{n}$ are homeomorphic.

Finally, we have to show that the sequence $T_{n}$ is equicontinuous. Suppose not. Then there exist $\epsilon^{*}>0$, a subsequence of $n$, and for $n$ in this subsequence $w_{n}, w_{n}^{\prime} \in A_{n}$ such that $\left|T_{n}\left(w_{n}\right)-T_{n}\left(w_{n}^{\prime}\right)\right|>\epsilon^{*}$ and either the distance in $A_{n}$ of $w_{n}$ and $w_{n}^{\prime}$ tends to 0 while $w_{n}$ and $w_{n}^{\prime}$ remain in a fixed square $Q_{a / 2}$ containing $F$ or else $w_{n}, w_{n}^{\prime}$ tend to $\infty$. We may assume $\epsilon^{*}<\phi(S) / 2$. Consider the first case. By Lemma 2 and (2.3) there exist $c>0$ and $n_{1} \geqq n_{0}$ such that $\psi\left(S_{n}, \epsilon^{*}\right) \geqq c$ for all $n \geqq n_{1}$. Choose $\epsilon<c / 20(\beta+1)$ small enough that any grating $\pi$ based on $Q_{a}$ with $\bar{\Delta}(\pi)<10 \epsilon a$ is fine relative to $A$ and $n \geqq n_{1}$ so that $w_{n}$ and $w_{n}^{\prime}$ are distant $\left\langle\eta_{1} a / 2\right.$. We construct again an $\epsilon \delta$ grating. This time not only lines in $\pi_{0}$ distant at most $\eta_{1} a$ from $F$ but also those distant in $A_{n}$ at most $\eta_{1} a$ from $w_{n}$ are deleted. Then $w_{n}$, $w_{n}^{\prime}$ belong to the same bounded component $Z$ of $A_{n}-K_{n}$; and if we suppose $c<\phi\left(S_{n}\right)$ (as we may) then $Z^{-}$is a 2-cell with relative boundary $\gamma$ a cross-cut or simple closed curve with diam $T_{n}(\gamma)<c$. Moreover, there exists $\lambda \in \Omega\left(A_{n}\right)$ contained in $A_{n}-Z^{-}$. Then

$$
\operatorname{diam} T_{n}\left(A_{n}-Z^{-}\right) \geqq \operatorname{diam} T_{n}(\lambda) \geqq \phi\left(S_{n}\right)>\epsilon^{*},
$$

and

$$
\operatorname{diam} T_{n}(Z) \geqq\left|T_{n}\left(w_{n}\right)-T_{n}\left(w_{n}^{\prime}\right)\right|>\epsilon^{*} .
$$

Hence, $\psi\left(S_{n}, \epsilon^{*}\right) \leqq \operatorname{diam} T_{n}(\gamma)<c$, a contradiction. 
In case $w_{n}$ and $w_{n}^{\prime}$ tend to $\infty$ we define $\gamma_{n}$ as in the first part of the proof so that diam $T_{n}\left(\gamma_{n}\right)<c$. For large $n, B_{n}$ contains some $\lambda \in \Omega\left(A_{n}\right)$ while $A_{n}-B_{n}^{-}$contains $w_{n}, w_{n}^{\prime}$. As before this is impossible.

\section{The main theorem.}

LEMma 7. Every polyhedron (not of the type of the 2-cell or 2-sphere) has a quasi-conformal representation on a normalized parallel slit domain.

This is a special case of mapping theorems for general Riemann domains proved in [3, Chapter II].

Lemma 8. Let $T$ be Dirichlet in a slit domain $A$ and $S$ the Fréchet surface represented by $T$. Then $L(S)=I(T, A)$; i.e., the Lebesgue area equals the classical area integral.

Proof. Let $\pi$ be a grating fine relative to $A$ such that $T$ represents a rectifiable curve on every arc of the associated set $K$. Now $K$ decomposes $A$ into nonoverlapping 2-cells $Z_{1}^{-}, \cdots, Z_{m}^{-}$disjoint from $A^{*}$ and annular regions $Y_{1}, \cdots, Y_{\nu}$ formed by 2 -cells $Z_{m+1}^{-}, \cdots, Z_{\bar{k}}$ which meet $A^{*}$. For $j=1, \cdots$, $\nu, Y_{j}$ is bounded by $C_{j}$ and a polygonal curve in $K$. For $i=1, \cdots, m Z_{i}$ is either a rectangle (possibly formed by joining two euclidean rectangles along an identified edge) or else contains $\infty$ or an inner vertex. In any case, $Z_{\vec{i}}$ is the image of a planar 2-cell $W_{i}$ under a one-one elementary transformation $\psi_{i}$, conformal except for one point at most. By known theorems, $T \psi_{i}$ is Dirichlet in $W_{i}$ and $I\left(T \psi_{i}, W_{i}\right)=I\left(T, Z_{i}\right), i=1, \cdots, m$. Then if $S_{i}$ is the surface represented by $\left(T, Z_{i}^{-}\right), L\left(S_{i}\right)=I\left(T, Z_{i}\right)$ by the known analogue of Lemma 11 for planar 2-cells [1, p. 44].

Given $\epsilon>0$ choose $\pi$ so that, writing $A_{\epsilon}=\bigcup_{i=1}^{m} Z_{i}^{-}$, we have $I(T, A)$ $-I\left(T, A_{\epsilon}\right)<\epsilon$ and diam $T\left(Z_{i}\right)<\epsilon / 2$ for $i=m+1, \cdots k$. If $S_{\epsilon}$ is that surface represented by $\left(T, A_{\epsilon}\right)$, then $L\left(S_{\epsilon}\right)=\sum_{i=1}^{m} L\left(S_{i}\right)$ by a result of Federer [6]. Moreover, $L\left(S_{\epsilon}\right) \leqq L(S)$ [5, (6.25)]. There is a homeomorphism $H_{\epsilon}$ of $A_{\epsilon}$ onto $A$ such that $H_{\epsilon}(w)=w$ if $w$ belongs to a slightly smaller subdomain interior to $A_{\epsilon}$ and $\left|T\left[H_{\epsilon}(w)\right]-T(w)\right|<\epsilon$ for all remaining $w \in A_{\epsilon}$. This shows that $\left\|S, S_{\epsilon}\right\|<\epsilon$. Hence, $\lim _{\epsilon \rightarrow 0} L\left(S_{\epsilon}\right)=L(S)$ since $L\left(S_{\epsilon}\right) \leqq L(S)$ and Lebesgue area is lower semi-continuous. However,

$$
L\left(S_{\epsilon}\right)=\sum_{i=1}^{m} L\left(S_{i}\right)=\sum_{i=1}^{m} I\left(T, Z_{i}\right)=I\left(T, A_{\epsilon}\right)
$$

and the right side tends to $I(T, A)$.

MAIN THEOREM. Every nondegenerate Fréchet surface $S$ of any finite topological type $\tau$ (not the type of the 2-cell or 2-sphere $\left({ }^{7}\right)$ ) with finite Lebesgue area has a quasi-conformal representation on a normalized slit domain.

(7) The theorem is true for 2-cells and 2-spheres provided we count the half plane and full plane with no slits as slit domains. This follows from Morrey's theorem [7]. 
Proof. Let $P_{n}$ be a sequence of polyhedra tending to $S$ such that $a\left(P_{n}\right)$ tends to $L(S)$. By Lemma 7 each $P_{n}$ has a quasi-conformal representation $T_{n}(u, v)$ on a normalized slit domain $A_{n} ; D\left(T_{n}, A_{n}\right)=a\left(P_{n}\right)$ is bounded.

We apply Lemma 6 . For any compact region $G$ disjoint from the slits of $A$, there is a further subsequence such that $T_{n}(u, v)$ converges to a limit $T(u, v)$ uniformly in $G$, by Ascoli's theorem. Diagonalizing, there is a still further subsequence, again denoted by $\left(T_{n}, A_{n}\right)$, independent of $G$ such that $T_{n}(u, v)$ tends to $T(u, v)$ uniformly in every such $G$. By equicontinuity, $T$ is continuous across slits of $A$ and at $\infty$. By known theorems [1, 32.7] $T(u, v)$ is absolutely continuous Tonelli in $G$ and $D(T, G) \leqq \lim \inf D\left(T_{n}, G\right)$ for every such $G$. This implies that $T$ is Dirichlet in $A$ and

$$
D(T, A) \leqq \lim \inf D\left(T_{n}, A_{n}\right)=\lim a\left(P_{n}\right)=L(S) .
$$

Let us show that $(T, A)$ represents $S$. For this we use Lemma 4 , and follow the notation there. Given $\epsilon>0$, we choose $\pi$ so fine that $\operatorname{diam} T(Z)<\epsilon / 3$ and $\operatorname{diam} T_{n}\left[H_{n}(Z)\right]<\epsilon / 3$ for any $n \geqq n(d)$ and component $Z$ of $A-K$. This is possible by equicontinuity and the remark that the diameter of every bounded component of $A_{n}-K_{n}$ (in the $A_{n}$ metric) is uniformly small with $\bar{\Delta}(\pi)$. For some $n_{1} \geqq n(d),\left|T_{n}\left(w_{j n}\right)-T\left(w_{j}\right)\right|<\epsilon / 3$ for all $n \geqq n_{1}$ and $j=1, \cdots, k$. Then $\left|T_{n}\left[H_{n}(w)\right]-T(w)\right|<\epsilon$ for all $w \in A, n \geqq n_{1}$. Since $\epsilon$ is arbitrary this shows that $(T, A)$ represents $\lim P_{n}$ which is $S$.

Finally, $L(S)=I(T, A)$ by Lemma 8 . Since $I(T, A) \leqq D(T, A)$ and $D(T, A) \leqq L(S)$, we have $D(T, A)=I(T, A)=L(S)$. Thus, $T$ is quasi-conformal.

8. Final remarks. In the above theorem slit domains can be replaced by domains of various other forms. For example, let $S$ be nondegenerate of genus 0 (i.e., of the type of a finitely connected planar Jordan region). Let $(T, A)$ represent $S$ quasi-conformally, $A$ a slit domain. There is a one-one conformal mapping $f$ of a planar region $J$ bounded by $\nu$ circles onto $A$ [3, p. 169]. It can be shown that $(T f, J)$ is also a quasi-conformal representation of $S$. For other possible classes of domains in case of genus not 0 , see [3, Chapters 4,5 ; $4]$.

AN EXAMPLE. Let $J$ be an annulus bounded by two concentric circles, and $g$ a segment joining opposite boundaries of $J$. One can construct a mapping $T(w)$ from $J$ into $E_{3}$ with the following properties: (1) $T$ is constant on $g$; (2) for $w_{1}, w_{2} \notin g, w_{1} \neq w_{2}$, we have $T\left(w_{1}\right) \neq T\left(w_{2}\right) \neq T(g)$; (3) given $w \notin g$ and $a>0$ there is a neighborhood $G$ of $g$ such that $T$ represents a curve of length $\geqq a$ on any curve joining $w$ and $G ;(4)(T, J)$ represents a surface $S$ of finite Lebesgue area. Intuitively, $S$ is an annulus in $E_{3}$ pinched together at a single point which cannot be approached on $S$ by any rectifiable curve. It is not difficult to show that $S$ has no Dirichlet representation, hence no quasiconformal representation. On the other hand, the fine cyclic decomposition $[2 ; 8]$ of $S$ has only one element. 
If $J$ is cut along $g$ and opposite sides of the cut are regarded as distinct, the resulting domain $J^{\prime}$ is a 2 -cell and $\left(T, J^{\prime}\right)$ represents a nondegenerate surface $S^{\prime}$ with the same Lebesgue area as $S$. By Morrey's theorem $S^{\prime}$ has a quasi-conformal representation. This sort of reasoning will be used in a subsequent paper to obtain representations of fine cyclic elements.

\section{REFERENCES}

1. L. Cesari, Surface area, Princeton University Press, 1956.

2. - Fine cyclic elements of surfaces of the type $\nu$, Riv. Mat. Univ. Parma. vol. 7 (1956) pp. 149-185.

3. R. Courant, Dirichlet's principle, New York, 1950.

4. J. Douglas, Minimal surfaces of higher topological structure, Ann. of Math. vol. 40 (1939) pp. 205-298.

5. H. Federer, On Lebesgue area, Ann. of Math. vol. 61 (1955) pp. 289-353.

6. - An addition theorem for Lebesgue area, Proc. Amer. Math. Soc. vol. 6 (1955) pp. 911-914.

7. C. B. Morrey, An analytic characterization of surfaces of finite Lebesgue area, Amer. J. Math. vol. 57 (1935) pp. 692-702 and vol. 58 (1936) pp. 313-332.

8. C. J. Neugebauer, B-sets and fine cyclic elements, Trans. Amer. Math. Soc. vol. 88 (1958) pp. 121-143.

9. T. Rad6, Length and area, Amer. Math. Soc. Colloquium Publications, 1948.

10. J. H. Roberts and N. E. Steenrod, Monotone transformations of two-dimensional manifolds, Ann. of Math. vol. 39 (1938) pp. 851-862.

11. M. Shiffman, The Plateau problem for minimal surfaces of arbitrary topological structure, Amer. J. Math. vol. 61 (1939) pp. 853-882.

12. L. C. Young, Some applications of the Dirichlet integral to the theory of surfaces, Trans. Amer. Math. Soc. vol. 64 (1948) pp. 317-355.

13. - On generalized surfaces of finite topological types, Memoirs Amer. Math. Soc., no. 17,1955 .

14. J. W. T. Youngs, The representation problem for Fréchet surfaces, Memoirs Amer. Math. Soc., no. 8, 1951 .

15. - Homeomorphic approximations to monotone mappings, Duke Math. J. vol. 15 (1948) pp. 87-94.

Purdue University,

LAFAYETTE, IND. 